\title{
Discrete Variable Methods for a Boundary Value Problem with Engineering Applications
}

\author{
By Riaz A. Usmani
}

\begin{abstract}
In this paper we develop numerical techniques of order 2, 4 and 6 for the solution of a fourth order linear equation. A priori error bound is obtained for the fourth order method to prove the convergence of the finite difference scheme. A sufficient condition guaranteeing the uniqueness of the solution of the boundary value problem is also given. Numerical illustrations are tabulated and results compared with the classical Runge-Kutta method.
\end{abstract}

1. Introduction. We consider the problem of bending a rectangular clamped beam of length $l$ resting on an elastic foundation. The vertical deflection $w$ of the beam satisfies the system

$$
\begin{gathered}
{[L+(k / D)] w=D^{-1} q(x), \quad L \equiv d^{4} / d x^{4},} \\
w(0)=w(l)=w^{\prime}(0)=w^{\prime}(l)=0,
\end{gathered}
$$

where $D$ is the flexural rigidity of the beam, and $k$ is the spring constant of the elastic foundation, and the load $q(x)$ acts vertically downwards per unit length of the beam. The details of the mechanical interpretation of (1.1) are given in [7, p. 175]. Mathematically, the system (1.1) belongs to a general class of boundary problems of the form

$$
\begin{gathered}
(L+f(x)) y(x)=g(x), \quad a<x<b, \\
y(a)=A_{1}, \quad y(b)=A_{2}, \quad y^{\prime}(a)=B_{1}, \quad y^{\prime}(b)=B_{2},
\end{gathered}
$$

where the functions $f(x)$ and $g(x)$ are continuous on $[a, b]$ and $A_{i}, B_{i}(i=1,2)$ are finite real arbitrary constants. The analytical solution of (1.2) for arbitrary choices of $f(x)$ and $g(x)$ cannot be determined. Faced with this difficulty, we resort to discrete variable methods for obtaining an approximate solution of the system. A numerical technique used for approximating $y$ over a finite set of grid points $\left\{x_{n}\right\} \subset[a, b]$ by finite difference methods is given in [1, p. 165].

We introduce the set $\left\{x_{n}\right\}$,

$$
x_{n}=a+n h, \quad h=(b-a)(N+1), \quad n=O(1) N+1 .
$$

We further define the discretization error $e_{n}=y_{n}-z_{n}$, where $y_{n}=y\left(x_{n}\right)$ and $z_{n}$ is its numerical approximation. The authors of [1] have proved

$$
\max \left[\max _{2 \leqslant n \leqslant N-1}\left|e_{n}\right|,\left|e_{1}\right| / h^{3 / 2},\left|e_{N}\right| / h^{3 / 2}\right] \leqslant O\left(h^{3 / 2}\right)
$$

provided $f(x) \geqslant 0$ on $[a, b]$. Babuska et al. thus showed that the resulting error is $O\left(h^{3 / 2}\right)$ based on the finite difference scheme.

Received September 16, 1977 ; revised January 27, 1978.

AMS (MOS) subject classifications (1970). Primary 65 L10.

Copyright $\odot$ 1978, American Mathematical Society 


$$
\left[\begin{array}{l}
\text { (i) }-4 y_{0}+7 y_{1}-4 y_{2}+y_{3}=2 h y_{0}^{\prime}+h^{4} y_{1}^{(4)}+O\left(h^{3}\right), \\
\text { (ii) } \delta^{4} y_{n}=h^{4} y_{n}^{(4)}+O\left(h^{6}\right), \quad n=2(1) N-1, \\
\text { (iii) } y_{N-2}-4 y_{N-1}+7 y_{N}-4 y_{N+1}=-2 h y_{N+1}^{\prime}+h^{4} y_{N+1}^{(4)}+O\left(h^{3}\right) .
\end{array}\right.
$$

They also remarked that in case of the boundary value problem (1.2) one may arrive at a more detailed analysis of the inverse matrix associated with the system of linear equations (1.4) in relation to the properties of the corresponding Green's function. For instance, such an analysis would yield $\max _{n}\left|e_{n}\right|=O\left(h^{2}\right)$.

The purpose of this note is two-fold. We first examine the conditions under which the system (1.2) has a unique solution. We then pass on to describe ways in which the numerical solution of (1.2) obtained in [1] can be improved. In fact, we develop and analyze three finite difference schemes of order 2,4 , and 6 , respectively, to obtain an approximate solution of (1.2). In the end numerical evidence is included to demonstrate the superiority and practical usefulness of our finite difference scheme for a wider class of boundary value problems (1.2) than considered in [1].

\section{Uniqueness of Solution of (1.2).}

THEOREM 2.1. The boundary value problem (1.2) has a unique solution if

$$
\inf _{x} f(x)=-\eta>-\sigma /(b-a)^{4},
$$

where $\sigma=500.5639 \ldots$

We preface the proof of this theorem by a lemma.

LEMMA 2.2. For the system

$$
(L-\lambda) y=0, \quad y(a)=y^{\prime}(a)=y(b)=y^{\prime}(b)=0,
$$

$\lambda \leqslant 0$ is not an eigenvalue; $\lambda=\lambda_{n}>0$ is an eigenvalue provided $\cos \left[(b-a) \lambda_{n}^{1 / 4}\right] \cosh \left[(b-a) \lambda_{n}^{1 / 4}\right]=1$.

The proof of the lemma is not difficult. We omit it for brevity. For corresponding eigenfunctions, see [7]. We remark that the equation $\cos \mu \cosh \mu=1$ has infinitely many roots

$$
\mu_{1}<\mu_{2}<\cdots<\mu_{n}<\cdots
$$

with $\mu_{n} \rightarrow \infty$ as $n \rightarrow \infty$. Also, two distinct roots lie in each one of the intervals

$$
((4 m-1) / 2,(4 m+1) / 2), \quad m=1,2, \ldots .
$$

We obtain numerically, using Newton-Raphson's method, the smallest root

$$
\mu_{1}=4.7300407 . .
$$

and $(b-a)^{4} \lambda_{1}=\mu_{1}^{4}=\sigma$ or $\lambda_{1}=\sigma /(b-a)^{4}$.

The proof of Theorem 2.1 now follows from Lemma 2.2 in the standard way.

3. Finite Difference Methods. Our basic finite difference formulas can be derived from

$$
h^{4} y_{n}^{\mathrm{iv}}=\left(\delta^{4}-\frac{1}{6} \delta^{6}+\frac{7}{240} \delta^{8}-\frac{41}{7560} \delta^{10}+\cdots\right) y_{n}, \quad n=2,3, \ldots,
$$


see [2, p. 19]. From (3.1) we obtain the scheme

(3.2) $\delta^{4} y_{n}=h^{4} y_{n}^{\text {iv }}+\frac{1}{6} h^{6} y^{(6)}\left(\xi_{n}\right), \quad n=2(1) N-1, x_{n-2}<\xi_{n}<x_{n+2}$.

We note that from (3.2), on neglecting truncation error, and setting $y_{n} \simeq z_{n}$, we obtain

$$
\delta^{4} z_{n}=h^{4} z_{n}^{\mathrm{iv}}, \quad n=2(1) N-1 .
$$

The system (3.3) gives us $N-2$ equations in the $N$ unknowns $z_{n}, n=1(1) N$. At the boundaries we develop the following formulas

$$
\text { (i) }-\frac{11}{2} y_{0}+9 y_{1}-\frac{9}{2} y_{2}+y_{3}=3 h y_{0}^{\prime}+\frac{h^{4}}{20}\left[-3 y_{0}^{\text {iv }}+18 y_{1}^{\text {iv }}\right]+\frac{7}{40} h^{6} y^{(6)}\left(\xi_{1}\right) \text {, }
$$

(ii) $y_{N-2}-\frac{9}{2} y_{N-1}+9 y_{N}-\frac{11}{2} y_{N+1}$

$$
=-3 h y_{N+1}^{\prime}+\frac{h^{4}}{20}\left[18 y_{N}^{\mathrm{iv}}-3 y_{N+1}^{\mathrm{iv}}\right]+\frac{7}{40} h^{6} y^{(6)}\left(\xi_{N}\right),
$$

where $x_{0}<\xi_{1}<x_{3}$ and $x_{N-2}<\xi_{N}<x_{N+1}$.

From (3.4), on neglecting truncation errors and setting $y_{n} \simeq z_{n}$, we obtain two more equations in the $z_{n}$ unknowns

(i) $-\frac{11}{2} z_{0}+9 z_{1}-\frac{9}{2} z_{2}+z_{3}=3 h B_{1}+\frac{h^{4}}{20}\left[-3 z_{0}^{\mathrm{iv}}+18 z_{1}^{\mathrm{iv}}\right]$,

(ii) $z_{N-2}-\frac{9}{2} z_{N-1}+9 z_{N}-\frac{11}{2} z_{N+1}=-3 h B_{2}+\frac{h^{4}}{20}\left[18 z_{N}^{\mathrm{iv}}-3 z_{N+1}^{\mathrm{iv}}\right]$, where $z_{n}^{\text {iv }}=-f_{n} z_{n}+g_{n}$.

Therefore, Eqs. 3.5(i), (3.3), and 3.5(ii) form our method of order 2. We shall give a general method for proving the convergence of our finite difference scheme in the next section. Note that the matrix associated with the system of linear equations in unknowns $z_{n}$ is a five-band matrix.

Our fourth order method is based on the formulas

(i) $-\frac{11}{2} y_{0}+9 y_{1}-\frac{9}{2} y_{2}+y_{3}$

$$
=3 h y_{0}^{\prime}+\frac{h^{4}}{280}\left[8 y_{0}^{\mathrm{iv}}+151 y_{1}^{\mathrm{iv}}+52 y_{2}^{\mathrm{iv}}-y_{3}^{\mathrm{iv}}\right]+\frac{h^{8}}{6720} y_{0}^{\mathrm{viii}}+O\left(h^{9}\right),
$$

(ii) $\delta^{4} y_{n}=\frac{h^{4}}{6}\left[y_{n-1}^{\mathrm{iv}}+4 y_{n}^{\mathrm{iv}}+y_{n+1}^{\mathrm{iv}}\right]-\frac{h^{8}}{720} y_{n}^{\mathrm{viii}}+O\left(h^{9}\right)$,

(iii) $y_{N-2}-\frac{9}{2} y_{N-1}+9 y_{N}-\frac{11}{2} y_{N+1}$

$$
\begin{aligned}
= & -3 h y_{N+1}^{\prime}+\frac{h^{4}}{280}\left[-y_{N-2}^{\mathrm{iv}}+52 y_{N-1}^{\mathrm{iv}}+151 y_{N}^{\mathrm{iv}}+8 y_{N+1}^{\mathrm{iv}}\right] \\
& +\frac{h^{8}}{6720} y_{N+1}^{\mathrm{viii}}+O\left(h^{9}\right) .
\end{aligned}
$$


The derivation of these formulas can be effected by standard methods given in [1], [4]. However, 3.6(ii) can be very easily deduced from (3.1) by operating on both sides with $\left(1+\alpha \delta^{2}\right)$ and choosing $\alpha$ so that the coefficient of $\delta^{6}$ is zero and finding the coefficient of $\delta^{8}$ to be $1 / 720$. (This technique was pointed out to the author by Professor L. Fox, Oxford University, United Kingdom.)

Finally, our sixth order method is based on the scheme

(i) $-\frac{11}{2} y_{0}+9 y_{1}-\frac{9}{2} y_{2}+y_{3}=3 h y_{0}^{\prime}+h^{4} \sum_{n=0}^{5} \beta_{n} y_{n}^{\text {iv }}-\frac{1}{960} h^{10} y^{x}\left(\xi_{1}\right)$, $x_{0}<\xi_{1}<x_{3}$

(ii) $\delta^{4} y_{n}=\frac{h^{4}}{720}\left[-y_{n-2}^{\mathrm{iv}}+124 y_{n-1}^{\mathrm{iv}}+474 y_{n}^{\mathrm{iv}}+124 y_{n+1}^{\mathrm{iv}}-y_{n+2}^{\mathrm{iv}}\right]$

$$
+\frac{h^{10}}{3024} y^{x}\left(\xi_{n}\right), \quad x_{n-2}<\xi_{n}<x_{n+2}, n=2(1) N-1 \text {, }
$$

(iii) $y_{N-2}-\frac{9}{2} y_{N-1}+9 y_{n}-\frac{11}{2} y_{N+1}=-3 h y_{N+1}^{\prime}+h^{4} \sum_{n=0}^{5} \beta_{5-n} y_{N-2+n}^{\mathrm{iv}}$

$$
-\frac{1}{960} h^{10} y^{x}\left(\xi_{N}\right), \quad x_{N-2}<\xi_{N}<x_{N+1}
$$

where

$$
\left(\beta_{0}, \beta_{1}, \ldots, \beta_{5}\right)=\frac{1}{33600}(937,18240,5990,140,-135,28)
$$

The equation 3.7(ii) can also be deduced from (3.1) by operating on both sides of it with $\left(1+\alpha \delta^{2}+\beta \delta^{4}\right)$, choosing $\alpha, \beta$ so that the coefficients of $\delta^{6}$ and $\delta^{8}$ are both zero, and finding the coefficient of $\delta^{10}$ to be $1 / 3024$.

4. Convergence of our Numerical Methods. Let $Y=\left(y_{n}\right), Z=\left(z_{n}\right), C=\left(c_{n}\right)$, $T=\left(t_{n}\right), E=\left(e_{n}\right)$ be $N$-dimensional column vectors. Then we can write the standard matrix equations for any of the three numerical methods described in the previous section as follows:

(i) $M Y=C+T$,

(ii) $M Z=C$,

(iii) $M E=T$.

We also have in each of the three cases

$$
M=A+h^{4} B F, \quad F=\operatorname{diag}\left(f_{n}\right),
$$

where $A=\left(a_{m n}\right), A^{-1}=\left(a_{m n}^{*}\right)$ with $a_{11}=a_{N N}=9, a_{12}=a_{N, N-1}=-9 / 2$; otherwise, 


$$
a_{m n}=\left\{\begin{aligned}
6, & m=n \\
-4, & |m-n|=1 \\
1, & |m-n|=2 \\
0, & |m-n|>2
\end{aligned}\right.
$$

It is known [8] that $A$ is a monotone matrix and

$$
\left\|A^{-1}\right\|=\max _{m} \sum_{n} a_{m n}^{*} \leqslant \frac{(N+1)^{4}}{384}\left[1+\frac{8}{(N+1)^{3}}\right],
$$

see Appendix for proof.

Our main purpose is to derive a bound on $\|E\|=\max _{n}\left|e_{n}\right|$. In order to accomplish this we need the following lemma.

LEMMA 4.1. If $B$ is a matrix of order $N$ and $\|B\|<1$, then $(I+B)^{-1}$ exists and $\left\|(I+B)^{-1}\right\|<1 /(1-\|B\|)$.

For proof see [3, inequality (3.6.9)] .

We now turn back to the error equation 4.1 (iii) and write it in the form

$$
E=M^{-1} T=\left(A+h^{4} B F\right)^{-1} T=\left(I+h^{4} A^{-1} B F\right)^{-1} A^{-1} T,
$$

$$
\|E\| \leqslant \frac{\left\|A^{-1}\right\|\|T\|}{1-h^{4}\left\|A^{-1}\right\|\|B\|\|F\|} \text { by Lemma 4.1 }
$$

provided

$$
h^{4}\left\|A^{-1}\right\|\|B\|\|F\|<1 .
$$

For instance, for our numerical procedure based on (3.6), we have

$$
\begin{aligned}
& \|T\|<0.002183 h^{8} M_{8}, \quad M_{8}=\underset{x}{\max _{x}}\left|y^{(8)}(x)\right|, \\
& B=\left[\begin{array}{rrrrrrr}
\frac{151}{280} & \frac{13}{70} & -\frac{1}{280} & & & \\
\frac{1}{6} & \frac{2}{3} & \frac{1}{6} & & & & \\
& \frac{1}{6} & \frac{2}{3} & \frac{1}{6} & \ldots & & \\
& & \ldots & \ldots & \ldots & \\
& & & & \frac{1}{6} & \frac{2}{3} & \frac{1}{6} \\
& & & & & \\
& & & -\frac{1}{280} & \frac{13}{70} & \frac{151}{280}
\end{array}\right],
\end{aligned}
$$

so that $\|B\| \leqslant 1,\|F\|=\max _{x}|f(x)|=f_{M}$, and

$$
\|E\| \leqslant K h^{4},
$$

where 


$$
K=0.002183 M_{8} G\left(1-f_{M} G\right)^{-1}, \quad G=\frac{(b-a)^{4}}{384}+\frac{h^{3}(b-a)}{48},
$$

provided $f_{M}$ satisfies

$$
f_{M}=|f(x)|<\frac{384}{(b-a)^{4}+8 h^{3}(b-a)} .
$$

While stronger results of the form (4.7) no doubt exist, the upper bound in (4.7) cannot be disregarded altogether. The inequality (4.6) proves the convergence of the method. In a similar manner we can prove the convergence of the method based on 3.5(i), (3.3) and 3.5(ii) and, finally, the convergence of the method based on Eqs. (3.7).

5. Numerical Illustrations. In this section we illustrate the three numerical techniques discussed in Section 3 by the following two boundary value problems of the type (1.2), with $f(x) \geqslant 0$.

$$
y^{\text {iv }}+4 y=1, \quad y(-1)=y(1)=0, \quad y^{\prime}(-1)=-y^{\prime}(1)=\frac{\sinh 2-\sin 2}{4(\cosh 2+\cos 2)}
$$

The analytical solution of $(5.1)$ is

$$
\begin{gathered}
y(x)=0.25[1-2[\sin 1 \sinh 1 \sin x \sinh x \\
+\cos 1 \cosh 1 \cos x \cosh x] /(\cos 2+\cosh 2)] . \\
y^{\mathrm{iv}}+x y=-\left(8+7 x+x^{3}\right) e^{x} \\
y(0)=y(1)=0, \quad y^{\prime}(0)=1, \quad y^{\prime}(1)=-e .
\end{gathered}
$$

The analytical solution of $(5.2)$ is $y(x)=x(1-x) e^{x}$.

All computations are performed in double precision arithmetic using an IBM 370/65 computer at The University of Manitoba. The experiments are summarized in Table I.

To broaden the scope of the application of our methods, we now consider a boundary value problem of the form (1.2) with $f(x)$ changing sign on $[a, b]$. Consider, for instance

$$
\begin{gathered}
y^{\mathrm{iv}}-x y=-\left(11+9 x+x^{2}-x^{3}\right) e^{x}, \quad-1<x<1, \\
y(-1)=y(1)=0, \quad y^{\prime}(-1)=2 e^{-1}, \quad y^{\prime}(1)=-2 e,
\end{gathered}
$$

with $y(x)=\left(1-x^{2}\right) e^{x}$ as its analytical solution. We summarize the experimental results in Table II.

It is verified from Tables I and II that on reducing the step-size from $h$ to $h / 2$, the maximum observed error $\|E\|$ is approximately reduced by a factor $1 / 2^{p}$, where $p$ is the order of the numerical method, except possibly when the rounding errors are significant. Such entries in these tables have been underlined. 
DISCRETE VARIABLE METHODS

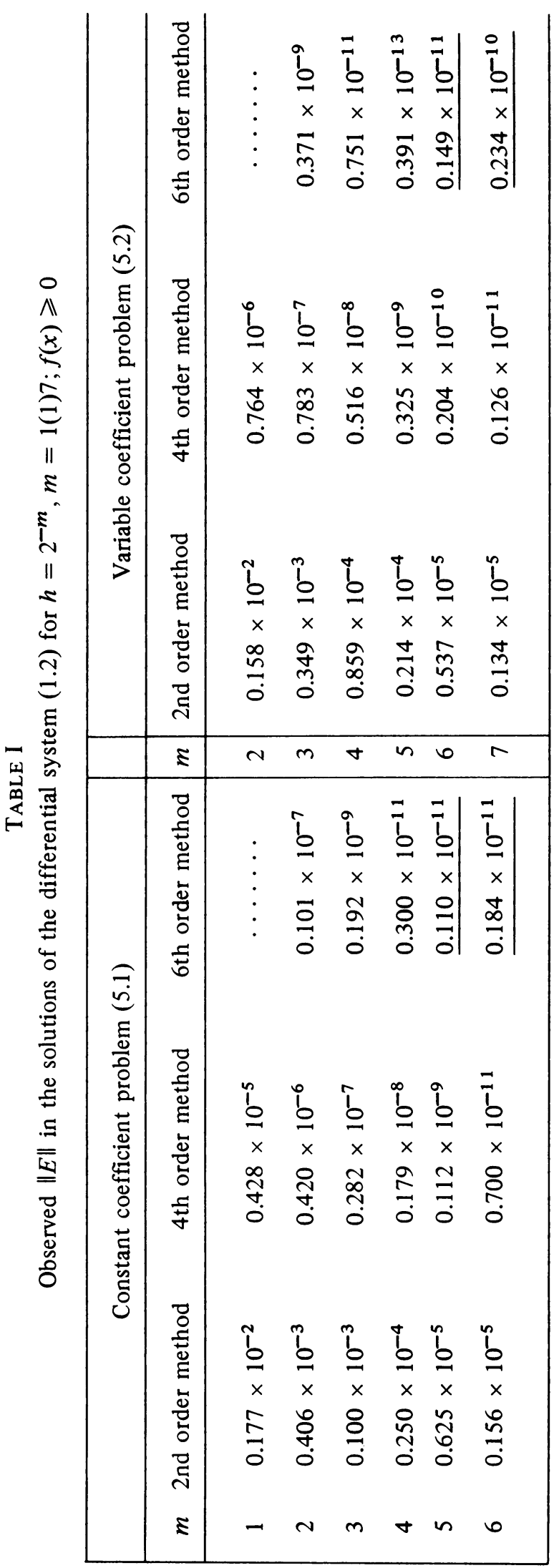


TABLE II

Observed $\|E\|$ for the problem (5.3), $f(x)$ changes sign, $h=2^{-m}$

\begin{tabular}{|c|c|c|}
\hline$m$ & 4 th order method & 6 th order method \\
\hline 1 & $0.128 \times 10^{-3}$ & $\cdots \cdots$ \\
\hline 2 & $0.127 \times 10^{-4}$ & $0.237 \times 10^{-6}$ \\
\hline 3 & $0.852 \times 10^{-6}$ & $0.494 \times 10^{-8}$ \\
\hline 4 & $0.543 \times 10^{-7}$ & $0.807 \times 10^{-10}$ \\
\hline 5 & $0.341 \times 10^{-8}$ & $0.156 \times 10^{-11}$ \\
\hline 6 & $0.303 \times 10^{-9}$ & $0.231 \times 10^{-10}$ \\
\hline 7 & $0.148 \times 10^{-8}$ & $0.351 \times 10^{-9}$ \\
\hline
\end{tabular}

For the sake of comparison we also solved some of these boundary value problems by a modified shooting technique, the details of which are given in [5]. It can be shown that a boundary value problem (1.2) is equivalent to a system of 12 first order differential equations. The details are omitted for brevity. The resulting system of differential equations is solved by a fourth order Runge-Kutta method [6, p. 110].

TABLE III

Observed $\|E\|$

\begin{tabular}{|c|c|c|c|}
\hline Problem & $h$ & $\begin{array}{l}\text { Runge-Kutta fourth order } \\
\text { method for modified } \\
\text { shooting technique }\end{array}$ & Our fourth order method \\
\hline$(5.1)$ & $\begin{array}{l}1 / 8 \\
1 / 16\end{array}$ & $\begin{array}{l}0.140 \times 10^{-5} \\
0.879 \times 10^{-7}\end{array}$ & $\begin{array}{l}0.282 \times 10^{-7} \\
0.179 \times 10^{-8}\end{array}$ \\
\hline$(5.2)$ & $\begin{array}{l}1 / 8 \\
1 / 16\end{array}$ & $\begin{array}{l}0.174 \times 10^{-4} \\
0.113 \times 10^{-5}\end{array}$ & $\begin{array}{l}0.783 \times 10^{-7} \\
0.516 \times 10^{-8}\end{array}$ \\
\hline
\end{tabular}

Table III shows that our fourth order method outperforms the classical fourth order Runge-Kutta method.

Acknowledgement. This research was supported by National Research Council of Canada.

The author also acknowledges the assistance of Mr. Marius Kung in providing the numerical results of Section 5 . 
Appendix. The inequality (4.3) is proved by Usmani and Meek in [8], but for the sake of completeness we shall outline the proof very briefly below. The matrix $A$ defined by (4.2) is of the form

$$
A=\left[\begin{array}{ccc}
9 & a^{T} & 0 \\
b & P_{N-2} & b^{R} \\
0 & a^{T R} & 9
\end{array}\right],
$$

where $a^{T}=(-9 / 2,1,0, \ldots, 0), b^{T}=(-4,1,0, \ldots, 0)$. Here $T$ denotes the operation of transposition and for a row vector $v=\left(v_{1}, v_{2}, \ldots, v_{n}\right), v^{R}=\left(v_{n}\right.$, $\left.v_{n-1}, \ldots, v_{1}\right)$. Note $a, b$ are $(N-2)$ dimensional column vectors. The matrix $P_{N-2}=\left(p_{i j}\right)$ is a five-band matrix of order $N-2$ such that

$$
p_{i j}=\left[\begin{array}{cl}
6, & i=j, \\
-4, & |i-j|=1, \\
1, & |i-j|=2, \\
0, & \text { otherwise. }
\end{array}\right.
$$

The matrix $A^{-1}$ is likewise of the form

$$
A^{-1}=\left[\begin{array}{ccc}
c & \alpha^{T} & d \\
\beta & Q_{N-2} & \beta^{R} \\
d & \alpha^{T R} & c
\end{array}\right],
$$

where $\alpha=\left(\alpha_{i}\right), \beta=\left(\beta_{i}\right)$ are $(N-2)$ dimensional column vectors and $c, d$ are scalars. Using $A A^{-1}=I_{N}$, we find that the unknowns $c, d, \alpha, \beta, Q_{N-2}$ satisfy the following equations.

$$
\begin{aligned}
& \text { (i) } 9 c+a^{T} \beta=1 \\
& \text { (ii) } 9 d+a^{T} \beta^{R}=0, \\
& \text { (iii) } c b+P_{N-2}^{\beta}+d b^{R}=0 \\
& \text { (iv) } 9 \alpha^{T}+a^{T} Q_{N-2}=0^{T}, \\
& \text { (v) } b \alpha^{T}+p_{N-2} Q_{N-2}+b^{R} \alpha^{T R}=I_{N-2} \text {. }
\end{aligned}
$$

Note $\mathbf{0}$ denotes a $(N-2)$ dimensional null column vector. The first three equations determine $c, d, \beta$ while the remaining two equations determine $\alpha, Q_{N-2}$. In each case we need to use $P_{N-2}^{-1}=\left(\bar{p}_{i j}\right)$ which is symmetric and for $i \geqslant j$

(5) $\bar{p}_{i j}=\frac{(N-i-1)(N-i) j(j+1)}{6 N(N-1)(N+1)}[i(j+2)(N-1)-(i+1)(j-1)(N+1)]$,

see [5]. Now a lengthy but elementary calculation yields

(6) $c=\frac{N^{2}}{3(N+1)^{2}} ; \quad d=\frac{N}{3(N+1)^{2}} ; \quad \beta_{i}=\frac{(i+1)(N-i)^{2}}{3(N+1)^{2}}, \quad i=1(1) N-2$. 
Now $\alpha$ is obtained on eliminating $Q_{N-2}$ from the remaining two equations in the form

$$
\begin{aligned}
\alpha_{i}=\{ & N(N-i-1)(N-i)[(3 N+1) i+2(N+1)] \\
& +i(i+1)[(3 N+1)(N-i-1)+2(N+1)]\} /\left[6(N-1)(N+1)^{3}\right], \\
& i=1(1) N-2 .
\end{aligned}
$$

Finally, from $4(v)$ we have

$$
Q_{N-2}=P_{N-2}^{-1}+H \quad \text { with } H=-P_{N-2}^{-1}\left(b \alpha^{T}+b^{R} \alpha^{T R}\right) .
$$

Let $H=\left(h_{i j}\right)$, then $h_{i j}$ is

$$
\begin{array}{r}
h_{i j}=\frac{(i+1)(N-i)}{6 N(N-1)(N+1)^{3}}\{(N-j-1)(N-j)(N-i)[(3 N+1) j+2(N+1)] \\
+j(j+1)(i+1)[(3 N+1)(N-j-1)+2(N+1)]\}, \\
i, j=1(1) N-2 .
\end{array}
$$

It is easy to conclude by inspection of $c, d, \alpha_{i}, \beta_{i}, \bar{p}_{i j}$ and $h_{i j}$ that the matrix $A$ is monotone (that is, $A^{-1}>0$ ). The sum of the elements of the $i$ th row of $P_{N-2}^{-1}$ is $i(i+1)(N-i-1)(N-i) / 24$ while the sum of the elements of the $i$ th row of $H$ is $(N-2)(N+3)(i+1)(N-i) /[24(N+1)]$. Finally, let the sum of the elements of the $i$ th row in $A^{-1}$ be $R_{i}$, then

$$
R_{i}=\frac{i(N-i+1)}{24(N+1)}[(N+1)(N-i+1) i+2], \quad i=1(1) N .
$$

It can be seen that $R_{i}$ attains a maximum value for $i=(N+1) / 2$. Thus,

$$
\left\|A^{-1}\right\| \leqslant R_{(N+1) / 2}=\frac{(N+1)(N+3)\left(N^{2}+3\right)}{384}
$$

and from this follows the desired inequality (4.3).

Department of Applied Mathematics
University of Manitoba
Winnepeg, Manitoba R3T 2N2, Canada

1. I. BABUSKA, M. PRAGER \& E. VITASEK, Numerical Processes in Differential Equations, Wiley (Interscience), New York, 1966.

2. L. FOX, The Numerical Solution of Two-Point Boundary Value Problems in Ordinary Differential Equations, Oxford Univ. Press, London and New York, 1957.

3. CARL-ERIK FRÖBERG, Introduction to Numerical Analysis, 2nd ed., Addison-Wesley, New York, 1969.

4. P. HENRICI, Discrete Variable Methods in Ordinary Differential Equations, Wiley, New York, 1962.

5. W. D. HOSKINS \& P. J. PONZO, "Some properties of a class of band matrices," Math. Comp., v. 26, 1972 , pp. 393-400.

6. W. RALSTON, Mathematical Methods for Digital Computers, Wiley, New York, 1960.

7. E. L. REISS, A. J. CALLEGARI \& D. S. AHLUWALIA, Ordinary Differential Equations with Applications, Holt, Rinehart and Winston, New York, 1976.

8. R. A. USMANI \& D. S. MEEK, "On the application of a five-band matrix in the numerical solution of a boundary value problem," Utilitas Math. (To appear.) 